\title{
On the Planetary Orbital Period Ratio Distribution In Multiple Planet Systems
}

\author{
Ji-Wei Xie (1,2 $^{1,-10}$ \\ ${ }^{1}$ Department of Astronomy \& Key Laboratory of Modern Astronomy and Astrophysics in \\ Ministry of Education, Nanjing University, 210093, China \\ ${ }^{2}$ Department of Astronomy and Astrophysics, \\ University of Toronto, Toronto, ON M5S 3H4, Canada \\ email: jwxie@astro.utoronto.ca
}

\begin{abstract}
Many multiple planet systems have been found by both radial velocity (RV) and transit surveys, such as the Kepler mission. Period ratio distribution of these planet candidates show that they do not prefer to be in or near Mean Motion Resonance (MMR). Nevertheless, there are small but significant excesses of candidate pairs both spaced slightly exterior to exact resonance, particular near the first order of MMR, such as 2:1 and 3:2. Here, we first review recent observational constraints on these multiple transiting systems and theoretical models, which attempt to understand their period ratio distributions. Then we identify a statistical effect based on an intrinsic asymmetry associated with MMR, and find it play an important role in shaping the period ratio distribution near MMR. Last but least, we also find such an intrinsic asymmetry is existing in asteroids of our solar system.
\end{abstract}

Keywords. celestial mechanics - planets and satellites: general

\section{Introduction}

\subsection{Core Observations: Excesses Exterior to $M M R$}

The Kepler mission has discovered from its first 16 months data over 2300 planetary candidates, which is over four times of the total number of extrasolar planets that were know in the past twenty years before its launch (Borucki et al. 2011, Batalha et al. 2013). Over one third $(>800)$ of these candidates are multiple transiting candidate planetary systems, and one remarkable feature of them, as shown by Lissauer et al. (2011) and Fabrycky et al. (2012), is that the vast majority of candidate pairs are neither in nor near low-order mean motion resonance (MMR hereafter, see also in Veras \& Ford 2012), however there are small but significant excesses of candidate pairs both spaced slightly exterior to exact resonance (or nominal resonance center), particularly near the first order of MMR, such as 2:1 and 3:2 (see the top panel of Fig. 1). In addition, the planets detected using radial velocity method (RV planets) reveal some similar excesses exterior to 2:1 MMR (see the bottom panel of Fig. 1).

\subsection{Other Related Observational Constraints}

Orbital eccentricities. By statistically studying the transit duration distribution of Kepler planet candidates, Moore et al. (2011) estimated their mean eccentricity at 0.1-0.25 for host stars with effect temperature less than $5100 \mathrm{~K}$. They also found a tendency that candidates in multiple transiting systems seem to have smaller eccentricities. In addition, Kane et al. (2012) and Plavchan et al. (2014) found that smaller candidates tend to have smaller eccentricities. However, as cautioned by themselves, all their results might be affected by potential biases or systematic effects, particularly due to errors in the 
stellar parameters. On another way, by analyzing the transit timing variation (TTV) of a subsample of candidates near MMR, Lithwick et al. (2012) and Xie(2012a) found that most of them are likely to have eccentricities as small as a few percents.

Orbital inclinations The geometry of multiple transiting systems naturally requires a low relative inclination of transiting objects. Detailed modelling show the Kepler's most multiple transiting systems are well aligned within a few degrees (Fabrycky et al. 2012; Figueira et al. 2012 and Fang \& Margot 2012)

\subsection{Previous Theoretical Explanations}

The period ratio distribution as shown in figure 1, especially the excesses outside the resonance center, has stimulated a number of theorists recently, who developed different models to understand and interpret it. Lithwick \& Wu(2012), Batygin \& Morbidelli(2013) and Delisle et al. (2012) considered that such an asymmetric period distribution around MMR as an outcome of resonant couples undergo eccentricity damping during some dissipative evolution, such as tidal dissipation [see also in Terquem \& Papaloizou(2007) for a similar scenario]. On the other side, Rein(2012) interpret the Kepler's period ratio distribution as a result of the combination of stochastic and smooth planet migration.
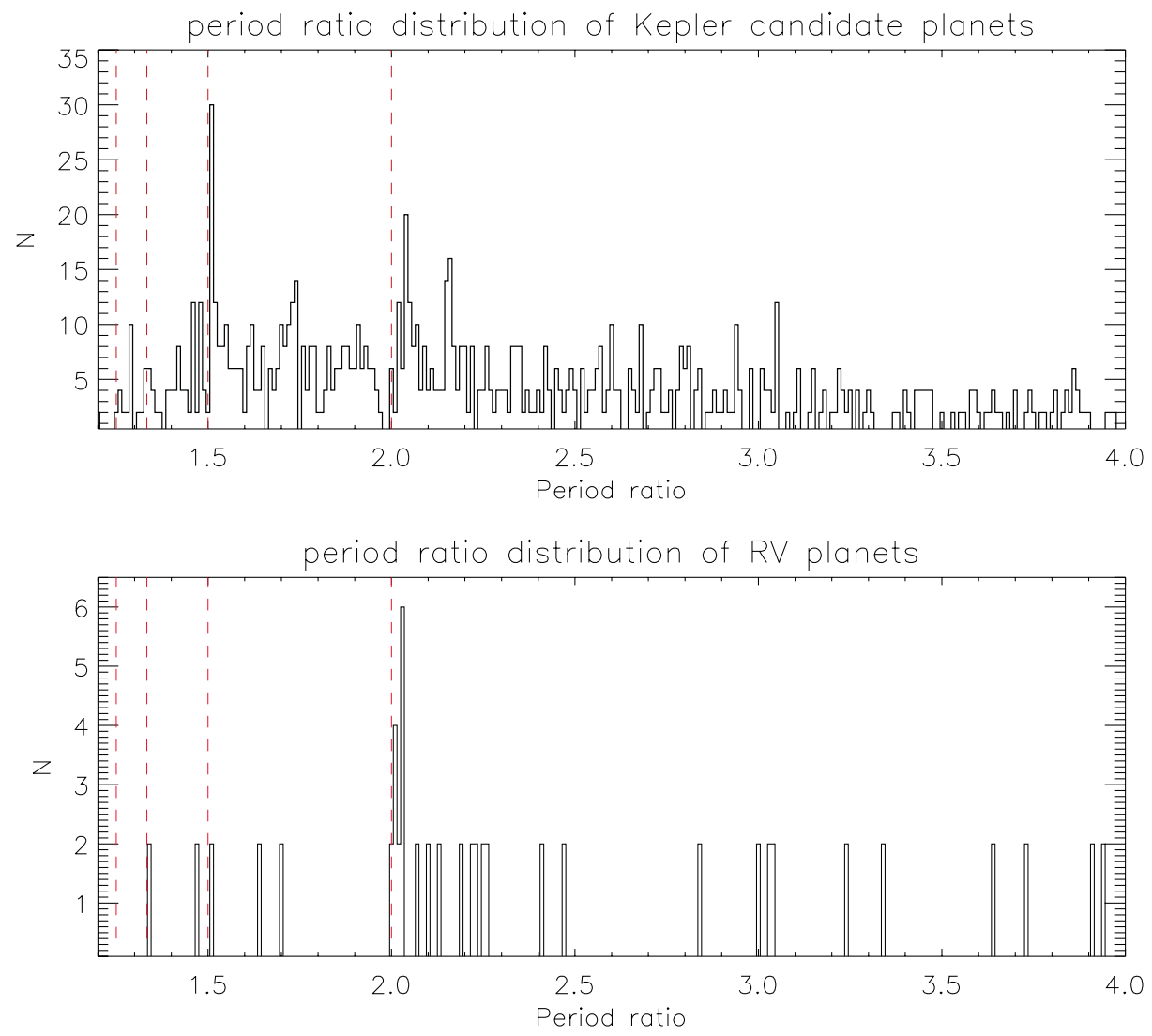

Figure 1. Period ratio distribution of Keplar's multiple transiting candidates planets (top, data from Batalha et al. 2013) and RV planets in multiple planet systems (bottom, data from "http://exoplanets.org/"). Bin sizes are 0.01. Vertical dashed lines indicate the center of $2: 1$, 3:2, 4:3 and 5:4 MMR. As can be seen, RV sample has a significant excess just exterior to 2:1 MMR while the Kepler sample has two significant excesses at 3:2 and 2:1 MMR. 

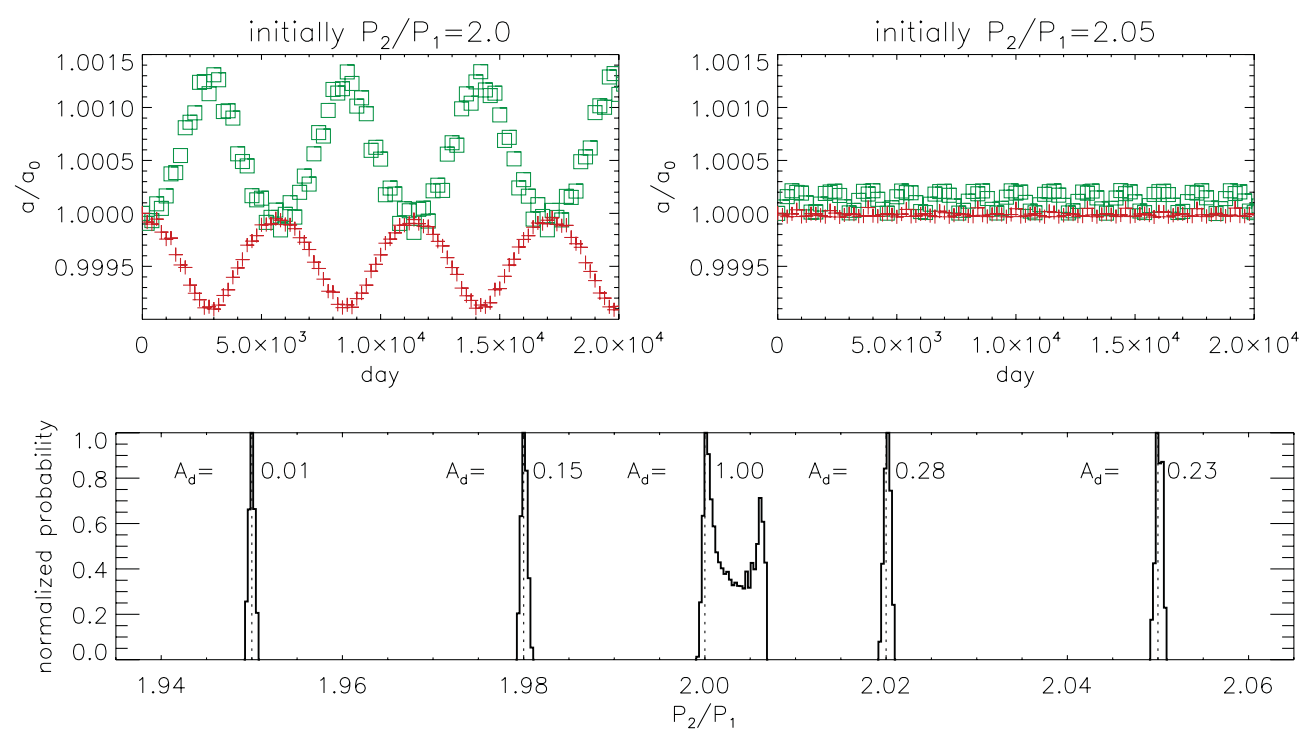

Figure 2. Evolutions of semimajor axes (top, normalized to the initial value, $a / a_{0}$ ) of two planets (red crosses for the inner planet and green boxes for the outer one) initially with orbital ratio of $p_{2} / p_{1}=2.0$ (top left panel) and $p_{2} / p_{1}=2.05$ (top right panel), circular $\left(e_{1}=e_{2}=0\right)$ and coplanar $\left(i_{12}=0\right)$ orbits. Performing 100 such experiments with random choices of planets' initial mean anomalies, we plot in the bottom wide panel the total orbital ratio (sampled at uniformly-spaced time points) distributions for the cases with initial $p_{2} / p_{1}=1.95,1.98,2.0,2.02$ and 2.05. Here we define the asymmetrical degree of the period ratio distribution, $A_{\mathrm{d}}$, as the ratio of the areas under the histogram that exterior and interior to the initial $p_{2} / p_{1}$ (A dot vertical line is plot in each histogram to separate these two areas).

In this communication, we identify an inherent asymmetric behaviour associated with MMR, which has been overlooked by previous studies. As we will show, such an inherent asymmetry induce a statistical effect on the period ratio, which can naturally explain the Kepler's excesses near 3:2 MMR (and partly for 2:1 MMR), providing a guide for further sophisticated models to fully understand the dynamics of these near-resonance pairs.

\section{A Statistical Interpretation}

\subsection{Intrinsic Asymmetry Near MMR}

We introduce the asymmetry from some simple planetary dynamical experiments as shown in Fig.2. We consider two planets with equal mass of $m_{1}=m_{2}=10 \mathrm{M}_{\oplus}$ in circular (i.e., orbital eccentricities $e_{1}=e_{2}=0$ ), coplanar (i.e., relative orbital inclination $i_{12}=0$ ), and strict $2: 1$ commensurable orbits (i.e., their orbital period ratios satisfy $p_{2} / p_{1}=2$ or their semimajor axes satisfy $a_{2} / a_{1}=2^{2 / 3}$ ) initially around a solar mass star (i.e., $M_{\star}=\mathrm{M}_{\odot}$ ). The location is set by $a_{1}=0.1 \mathrm{AU}$, which is motivated by hundreds of Kepler's candidate multiple transiting planets found in orbital periods of tens of days. We perform 100 simulations for the above initial conditions with all the other angular elements being set randomly $\dagger$. The typical results are shown in the top left panel of Fig.2,

$\dagger$ Although the stability depends on these angular elements Murray \& Dermott(1999), we didn't see any instability event, probably because of the low planetary mass, eccentricity and short evolution time adopted in our simulations 

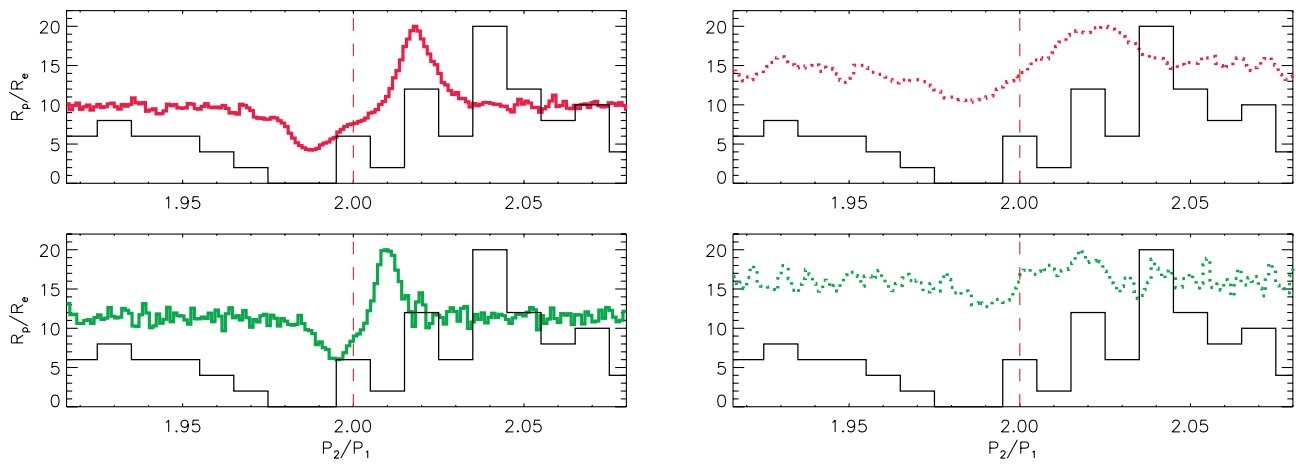

Asymmetry near 3:2 MMR
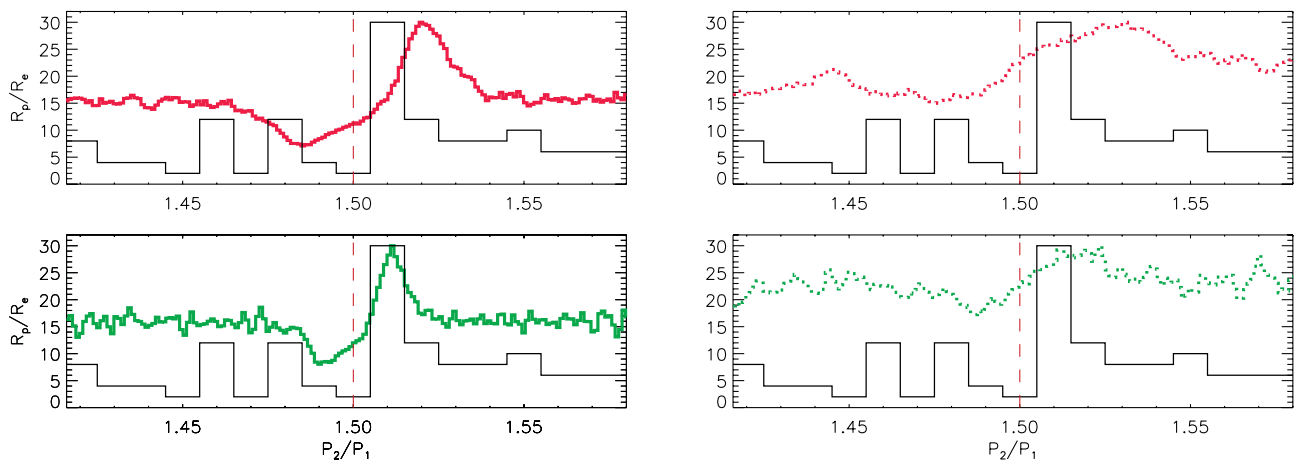

Figure 3. Orbital period ratio $\left(p_{2} / p_{1}\right)$ distributions: comparison between simulations (colourized curves, normalized to the same peak as the observational histogram) to Kepler's observations (black solid histograms) for planets near 2:1 resonance (top four panels) and those near 3:2 resonance (bottom four panels). For each panel above, we numerically integrate the orbital evolutions of a sample of 4000 planet pairs (the inner one always starting from an orbital semimajor axis $\left.a_{1}=0.1 \mathrm{AU}\right)$ with an uniform distribution of initial $p_{2} / p_{1}$ around the nominal resonance center (1.4-1.6 for 3:2 case, and 1.9-2.1 for 2:1 case), with equal mass (20 $M_{\oplus}$-green, $50 M_{\oplus}$-red), with a Rayleigh distribution of initial orbital eccentricity (from left to right: $\langle e\rangle=0.01$-solid and $\langle e\rangle=0.05$-dot), with a Rayleigh distribution of initial relative orbital inclination $(<i\rangle=2.5^{\circ}$ for all $)$, and with uniformly random distribution of all the other angular orbital elements.

and they are found to be independent of the initial choice of the two planets' orbital phases. As expected, the two planets' semimajor axes are periodically oscillating due to interchange of orbital energy and angular momentum. The key we want to emphasize here is an statistical asymmetry that the two planets always spend most time on orbits further away from each other, shifting their temporal average orbital ratio a little bit exterior to $2: 1$ (see the middle histogram in the bottom panel of Fig.2).

The asymmetry is found to occurs only at very close to (or in) nominal resonance center (here $p_{2} / p_{1}$ should be close to 2 ). If the initial orbital ratio is $p_{2} / p_{1}=2.05$, as shown in the right top panel and the right histogram in the bottom panel, then there is almost no such an asymmetry. Furthmore, we find strong dependences of this asymmetry on planets' masses $\left(m_{1}, m_{2}\right)$, orbital eccentricities $\left(e_{1}, e_{2}\right)$, and relative inclination $\left(i_{12}\right)$. In general [see Xie (2014) for a detailed results], this asymmetry requires $i_{12}<\sim 10^{\circ}$ (as mentioned in $\S 1.2$, this is well satisfied for Kepler candidates), and it become more significant for more massive planets initially with less eccentric orbits, but is nearly independent on the mass ratio of the planet pair. 

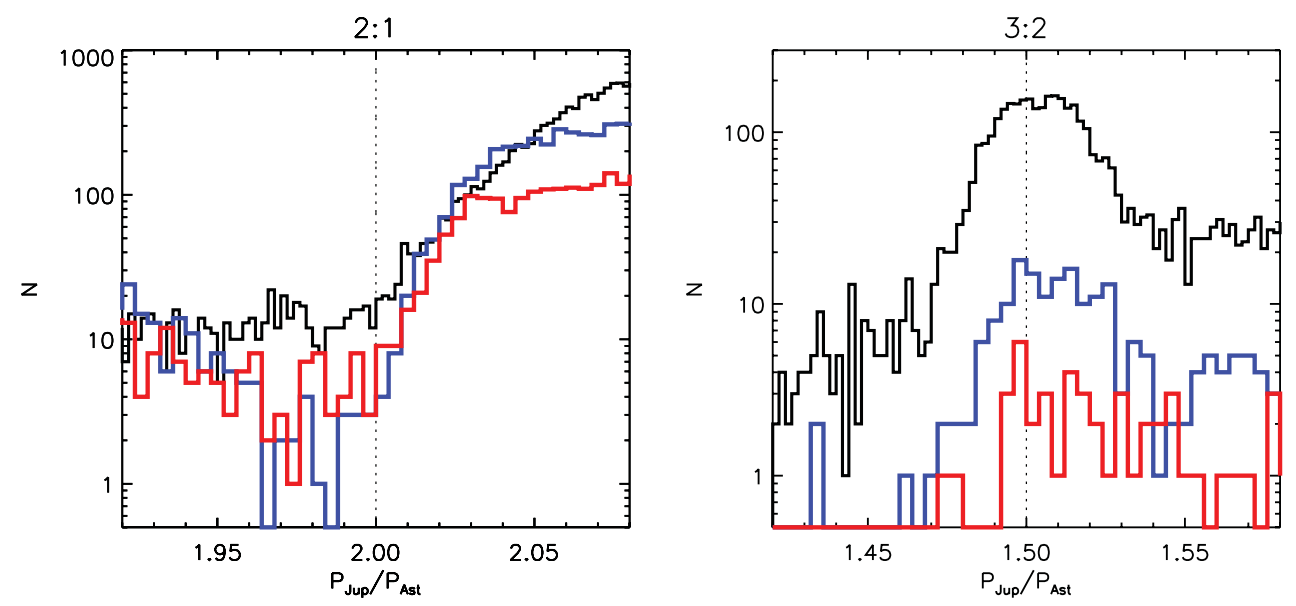

Figure 4. Distribution of number of asteroids around 2:1 and 3:2 MMR with Jupiter. $\mathrm{X}$ axis is the orbital period ratio between Jupiter and asteroids. In each panel, three histograms represent three subsamples with asteroid eccentricities in three different ranges (from top to bottom: black $=e>0.1$, blue $=0.05<e<0.1$ and red $=e<0.05)$. As can be seen, as their eccentricities decrease, the distribution become more asymmetrical with respect to the resonance center; more asteroids prefer to reside on the right side of the center. The data for plotting this figure are obtained from "http://www.minorplanetcenter.org/".

The above asymmetrical phenomenon is a little bit surprising to us at the first glance. In fact, as shown in Xie (2014), it is an natural result for the two near-resonance planets to conserve their energy and angular momentum.

\subsection{Applying to Kepler Data}

The asymmetrical $p_{2} / p_{1}$ distribution shown in Fig.2 are only due to pairs with initial $p_{2} / p_{1}$ exactly equal to the nominal resonance center. If starting many pairs with different initial $p_{2} / p_{1}$ around the center and add up their individual $p_{2} / p_{1}$ distributions, we then get the distribution of group $p_{2} / p_{1}$, which give us the final probability at each $p_{2} / p_{1}$. However, the issue is what initial $p_{2} / p_{1}$ distribution should be. Here we nominaly assume a flat initial $p_{2} / p_{1}$ distribution, and plot in Fig.3 their group $p_{2} / p_{1}$ distributions to compare to the $p_{2} / p_{1}$ distributions given by current Kepler candidates.

- For the 3:2 case, as shown in bottom left of Fig.3, the observational $p_{2} / p_{1}$ distribution can be generally reproduced with mean eccentricity no more than $1 \%$ and typical planetary mass of $20 \mathrm{M}_{\oplus}$. Such an mass range is acceptable given the planetary radii range of $\sim 2-4 R_{\oplus}$. The fitting is pretty good near the resonance center but worse far way from the center. This may be probably due to the small observation data there and/or the assumption of flat initial $p_{2} / p_{1}$ brakes down as the range of $p_{2} / p_{1}$ extends.

- For the 2:1 case, the observation also shows an evident pileup, not so sharp as in the 3:2 case but more extended exterior to the resonance center. As shown in Fig.3, neither the models with planetary mass of 20 nor $50 \mathrm{M}_{\oplus}$ can fit the observation. Although we can find a much better fit by increasing the planetary mass, e.g., to $100 \mathrm{M}_{\oplus}$, we doubt it because first, such an mass seems too high for the candidates near 2:1 with radii in a range of $\sim 2-4 R_{\oplus}$, and furthermore, the observation show an evident gap interior to the 2:1 resonance center, which cannot be reproduced in any simulated case. Thus, we feel that there may be some other unknown mechanisms which partly contribute the period ratio distribution near 2:1 (especially the gap). 


\subsection{Evidence from Asteroid Belt}

We also find some evidences of such intrinsic asymmetry from the asteroid belt. As shown in Fig.4, the period ratio (between that of Jupiter and asteroids) distribution become more asymmetrical (the side exterior to MMR center is favoured) as the asteroid eccentricities decrease. This statistical phenomenon is predicted by the intrinsic asymmetry associated with MMR. It is worthy noting that such a statistical effect differs from the statistical hypothesis attempting to explaining the Kirkwood gaps (see reviews by Scholl 1979 and Moons 1996).

\section{Conclusions}

Both Kepler and RV planets (Fig. 1) show asymmetrical period ratio distribution around MMR. Before looking for sophisticated models to understand the underlying physics and dynamics of this feature, one has to be sure to what degree it can be explained by itself with least assumptions. We identify an intrinsic asymmetry associated with MMR, which itself alone can recover the major feature of the observed period ratio distribution, especially around 3:2 MMR. Nevertheless, we caution that some other mechanisms are needed to fully understand the observation, especially the part around 2:1 MMR. The effect of the intrinsic asymmetry identified here is also seen in the asteroids of our solar system.

\section{References}

Batalha, N. M., Rowe, J. F., Bryson, S. T., et al. 2013, ApJS, 204, article id. 24

Batygin, K. \& Morbidelli, A. 2013, AJ, 145, article id.1

Borucki, W. J., Koch, D. G., Basri, G., et al. 2011, ApJ, 736, 19

Delisle, J.-B., Laskar, J., Correia, A. C. M., \& Boué, G. 2012, A $\mathscr{J} A$, 546, id.A71

Fabrycky, D. C., Lissauer, J. J., Ragozzine, D., et al. 2012, arXiv:1202.6328

Fang, J. \& Margot, J.-L. 2012, ApJ, 761, article id. 92

Figueira, P., Marmier, M., Boué, G., et al. 2012, A\&A, 541, A139

Kane, S. R., Ciardi, D. R., Gelino, D. M., \& von Braun, K. 2012, MNRAS, 425, 757

Lissauer, J. J., Ragozzine, D., Fabrycky, D. C., et al. 2011, ApJS, 197, 8

Lithwick, Y. \& Wu, Y. 2012, ApJL, 756, L11

Lithwick, Y., Xie, J., \& Wu, Y. 2012, ApJ, 761, article id. 122

Moons, M. 1996, CeMDA, 65, 175

Moorhead, A. V., Ford, E. B., Morehead, R. C., et al. 2011, ApJS, 197, 1

Murray, C. D. \& Dermott, S. F. 1999, Solar System Dynamics (Cambridge University Press)

Plavchan, P., Bilinski, C., \& Currie, T. 2014, PASP, 126, 34

Rein, H. 2012, MNRAS, 427, L21

Scholl, H. 1979, Dynamics of the Solar System, 81, 217

Terquem, C. \& Papaloizou, J. C. B. 2007, ApJ, 654, 1110

Veras, D. \& Ford, E. B. 2012, MNRAS, 420, L23

Xie, J.-W. 2013a, ApJS, 208, article id.22

Xie, J.-W. 2014, ApJS, 210, article id. 25 